\title{
Commentary: The Fontan paradox: A de Leval challenge
}

\author{
Tain-Yen Hsia, MD, MSc
}

\footnotetext{
From the Department of Pediatric Cardiac Surgery, Yale New Haven Children's Hospital, Yale School of Medicine, New Haven, Conn.

Disclosures: Author has nothing to disclose with regard to commercial support.

Received for publication April 22, 2019; accepted for publication April 22, 2019; available ahead of print May 23, 2019

Address for reprints: Tain-Yen Hsia, MD, MSc, Department of Pediatric Cardiac Surgery, Yale School of Medicine, PO Box 208064, New Haven, CT 06520-8064 (E-mail: tain-yen.hsia@yale.edu).

J Thorac Cardiovasc Surg 2019;158:1434-5

$0022-5223 / \$ 36.00$

Copyright (c) 2019 by The American Association for Thoracic Surgery

https://doi.org/10.1016/j.jtcvs.2019.04.066
}

Amongst his innumerable pioneering contributions to the field of congenital heart surgery, Marc de Leval's careerlong fascination with the Fontan circulation transformed the way we perform the operation and has continued to influence our understanding of this singular physiology. From recognizing the futility of incorporating the right atrium as part of the Fontan circulation to opening our minds to the power of computers as tools to optimize flow dynamics, de Leval has always asked questions others lacked the imagination to ask and has answered them through ingenuity and industry. Among the nearly 50 articles de Leval has authored or coauthored relating to the Fontan circulation lies a wonderfully insightful 2005 review. ${ }^{1}$ In a wide-ranging discourse on the evolution and effects of the Fontan circulation, de Leval coined the term Fontan paradox to describe the unique physiologic consequence of systemic venous hypertension coexisting with pulmonary arterial hypotension. At the very end of his dissertation, de Leval postulated that a device capable of producing a step down in vena caval pressure of $5 \mathrm{~mm} \mathrm{Hg}$ and a step up in pulmonary arterial pressure of $5 \mathrm{~mm} \mathrm{Hg}$ could potentially reverse the Fontan paradox.

Lin and colleagues ${ }^{2}$ introduce a novel design to take up the de Leval challenge. They propose the placement of a multilumen cannula in the superior vena cava to assist a failing Fontan circulation by drawing systemic venous blood into an external centrifugal pump and returning (or discharging) it to the branch pulmonary arteries. The authors test this concept through computational fluid dynamics (CFD) simulations, using idealized and patientspecific models, to demonstrate a potential pressure gain of 4 to $9 \mathrm{~mm} \mathrm{Hg}$ in pulmonary arterial circulation. Their computations additionally show acceptable simulated hemolysis and shear stress levels. Although the proposed design exists only in the authors' imaginations and within the confines of CFD software, they already propose percutaneous strategies to place discharge cannulas into the individual pulmonary arteries. Although the authors should be applauded for thinking outside the box, several caveats

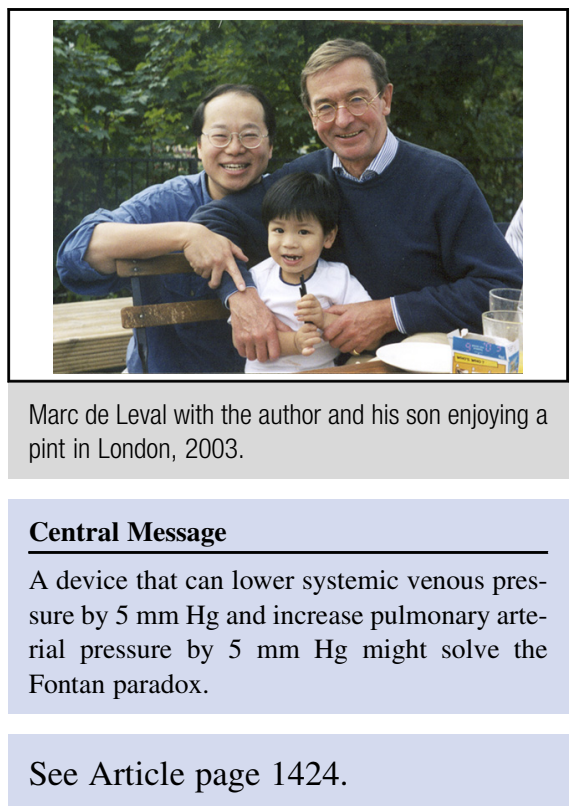

require further elucidation. Because their concept is not too dissimilar to that of venovenous extracorporeal membrane oxygenation without the oxygenator, the present design would likely only be suitable for short-term, transitional support, perhaps as a bridge to heart transplantation. Moreover, the proposed device would not ameliorate a failing Fontan circulation where ventricular dysfunction is the causative mechanism. Lastly, the presence of a large cannula in the superior vena cava could cause obstruction to the superior venous return that might further complicate a failing Fontan circulation.

Initially developed in the 1940 s and 1950 s to study problems in aeronautics such as airflow around a foil, CFD has become a ubiquitous tool to solve and analyze physical problems that involve fluid flow. Indeed, the Food and Drug Administration has increasingly mandated the integration of computational modeling in the evaluation and testing processes for new devices. ${ }^{3}$ Therefore, the adaptation of CFD as a platform to evaluate novel cardiovascular surgical concepts or devices is logical, especially when exploration in human subjects would not be ethically or technically feasible. However, one has to understand the limitations of CFD as a platform to mimic the human circulation. In addition to its inability to incorporate inherent biological processes such as thrombosis and hemolysis, CFD requires specific boundary conditions and assumptions to solve the governing fluid dynamics equations. In the present study, the authors chose fixed flow at the inlets (superior and inferior vena cava) and fixed pressures at the outlets (right 
and left pulmonary arteries). Rather than a complete simulation of the entire circulatory system in a patient with Fontan physiology, their model is an isolated, open-circuit model of their region of interest; that is, the cavopulmonary junction. In reality, how blood flows through a cavopulmonary junction influences and is influenced by the rest of the cardiopulmonary circulation. In other words, our circulatory system, even when a ventricular pump is absent, is a closed loop with continuous variations in pressure and flow occurring at its every part. An isolated CFD model, however detailed and 3-dimensional patient-specific, will miss these important interactions and lead to answers that may be either inaccurate or insufficient or both. In the present scenario, what we would most like to know, but unfortunately is left unanswered, is the device's influence on cardiac output and vena caval pressures. Focusing only on the cavopulmonary junction, the authors cannot tell us if their Fontan-assist strategy leads to higher cardiac outputs and lower venous pressures. Thus, they have only answered half of de Leval's challenge.

Since 2002, the group led by de Leval and his collaborators in Milan, Italy, has pioneered the concept of multiscale modeling, which allows for fully closed loop simulations of the entire cardiopulmonary circulation in single ventricle physiology. ${ }^{4}$ By coupling a detailed CFD model of a specific region of interest, such as a stage 1 Norwood reconstruction or a cavopulmonary junction, with a mathematical description of the entire circulation (lumped parameter network), multiscale modeling continuously adjusts and provides (iteratively) the boundary conditions of the CFD, which outputs information that influences the rest of the circulation. This type of modeling can provide the sophistication needed to understand not only pressures and flows, but also the physiologic consequences of surgical technique or pharmacologic intervention, such as cerebral and systemic oxygen delivery. In the case of a Fontanassist device, multiscale modeling would have been able to predict the effects on cardiac output and central venous pressures.

In 2005, de Leval reminded us that William Harvey-the first person to fully describe the human circulation and to recognize that 2 ventricular chambers are compulsorywould be furious with our efforts to place the systemic venous return in series with the pulmonary circulation without the interposition of a ventricle. Having challenged Harvey throughout his career, de Leval also left a roadmap for us to find a way to reverse the Fontan paradox: A device that can lower vena caval pressure by $5 \mathrm{~mm} \mathrm{Hg}$ and increase pulmonary arterial pressure by $5 \mathrm{~mm} \mathrm{Hg}$. The question remains: Can we answer his challenge?

\section{References}

1. de Leval MR. The Fontan circulation: a challenge to William Harvey? Nat Clin Pract Cardiovasc Med. 2005;2:202-8.

2. Lin WCP, Doyle MG, Roche SL, Honjo O, Forbes TL, Amon CH. Computational fluid dynamic simulations of a cavopulmonary assist device for failing Fontan circulation. J Thorac Cardiovasc Surg. 2019;158:1424-33.e5.

3. Stewart SFC, Paterson EG, Burgreen GW, Hariharan P, Giarra M, Reddy V, et al Assessment of CFD performance in simulations of an idealized medical device: results of FDA's first computational interlaboratory study. Cardiovasc Engineer Technol. 2012;3:139-60.

4. Hsia TY, Figliola R. Multiscale modeling of single ventricle hearts for clinical decision support: a Leducq transatlantic network of excellence. Eur J Cardiothorac Surg. 2016;49:365-8. 